\title{
REVIEW
}

\section{Lipidomics of Alzheimer's disease: current status}

\author{
Paul L Wood*
}

\begin{abstract}
Alzheimer's disease (AD) is a cognitive disorder with a number of complex neuropathologies, including, but not limited to, neurofibrillary tangles, neuritic plaques, neuronal shrinkage, hypomyelination, neuroinflammation and cholinergic dysfunction. The role of underlying pathological processes in the evolution of the cholinergic deficit responsible for cognitive decline has not been elucidated. Furthermore, generation of testable hypotheses for defining points of pharmacological intervention in $\mathrm{AD}$ are complicated by the large scale occurrence of older individuals dying with no cognitive impairment despite having a high burden of AD pathology (plaques and tangles). To further complicate these research challenges, there is no animal model that reproduces the combined hallmark neuropathologies of AD. These research limitations have stimulated the application of 'omics' technologies in AD research with the goals of defining biologic markers of disease and disease progression and uncovering potential points of pharmacological intervention for the design of $A D$ therapeutics. In the case of sporadic AD, the dominant form of dementia, genomics has revealed that the $\varepsilon 4$ allele of apolipoprotein E, a lipid transport/ chaperone protein, is a susceptibility factor. This seminal observation points to the importance of lipid dynamics as an area of investigation in AD. In this regard, lipidomics studies have demonstrated that there are major deficits in brain structural glycerophospholipids and sphingolipids, as well as alterations in metabolites of these complex structural lipids, which act as signaling molecules. Peroxisomal dysfunction appears to be a key component of the changes in glycerophospholipid deficits. In this review, lipid alterations and their potential roles in the pathophysiology of AD are discussed.
\end{abstract}

\section{*Correspondence: paul.wood@|munet.edu}

Metabolomics Unit, Department of Pharmacology, DeBusk College of Osteopathic Medicine, Lincoln Memorial University, 6965 Cumberland Gap Parkway, Harrogate, TN 37752, USA

\section{Introduction}

There is no animal model of Alzheimer's disease (AD) that possesses the hallmark features of the disease: neurofibrillary tangles, neuritic plaques, neuroinflammation, neuronal shrinkage, hypomyelination and cholinergic dysfunction. This limitation to current AD research highlights the critical nature of the 'omics' technologies as tools to study AD patients and AD autopsy tissues. This review will focus on lipidomics findings in AD. The application of lipidomics to $\mathrm{AD}$ research is logical in that the brain is the most lipid-rich organ in the human body. In addition, genomics studies have consistently demonstrated that the $\varepsilon 4$ allele of apolipoprotein E (ApoE), a lipid transport/chaperone protein, is a susceptibility, but not obligatory, factor for late onset (sporadic) AD. Furthermore, the severe cerebral macrostructural atrophy that is a hallmark feature of AD must involve the loss of structural lipids. Lipidomics has clearly shown that two major structural lipid classes are decreased in AD. These are the glycerophospholipids and sphingolipids. The current status of lipidomics findings and the potential roles of these lipid deficits in the pathophysiology of $\mathrm{AD}$ will be discussed. This review complements recent reviews of lipidomics in $\mathrm{AD}$ [1-3].

\section{Glycerophospholipids}

\section{Ethanolamine glycerophospholipids}

Early reports [4] of decreased phospholipid content in AD white matter were soon followed by reports of decreased levels of the phospholipid precursor ethanolamine in AD brain [5,6], cerebrospinal fluid (CSF) [7] and plasma [7] and increased brain levels of the degradation product glycerophosphoethanolamine [6]. The first descriptions of decrements in ethanolamine plasmalogens (PlsEtns), relative to phosphatidylethanolamines in $\mathrm{AD}$ brain, were published in 1995 [8]. Plasmalogens are a subclass of glycerophospholipids that possess a vinyl ether fatty alcohol substituent at sn-1 of the glycerol backbone (Figure 1). The ether linkage at sn-1 is achieved by addition of a fatty alcohol to the glycerol backbone and is conducted solely in peroxisomes (Figure 2). Subsequent desaturation to form the vinyl ether linkage takes place in the endoplasmic reticulum (Figure 2). Decrements in PlsEtns were shown to be disease specific since they were not measured in Huntington's caudate nucleus or 


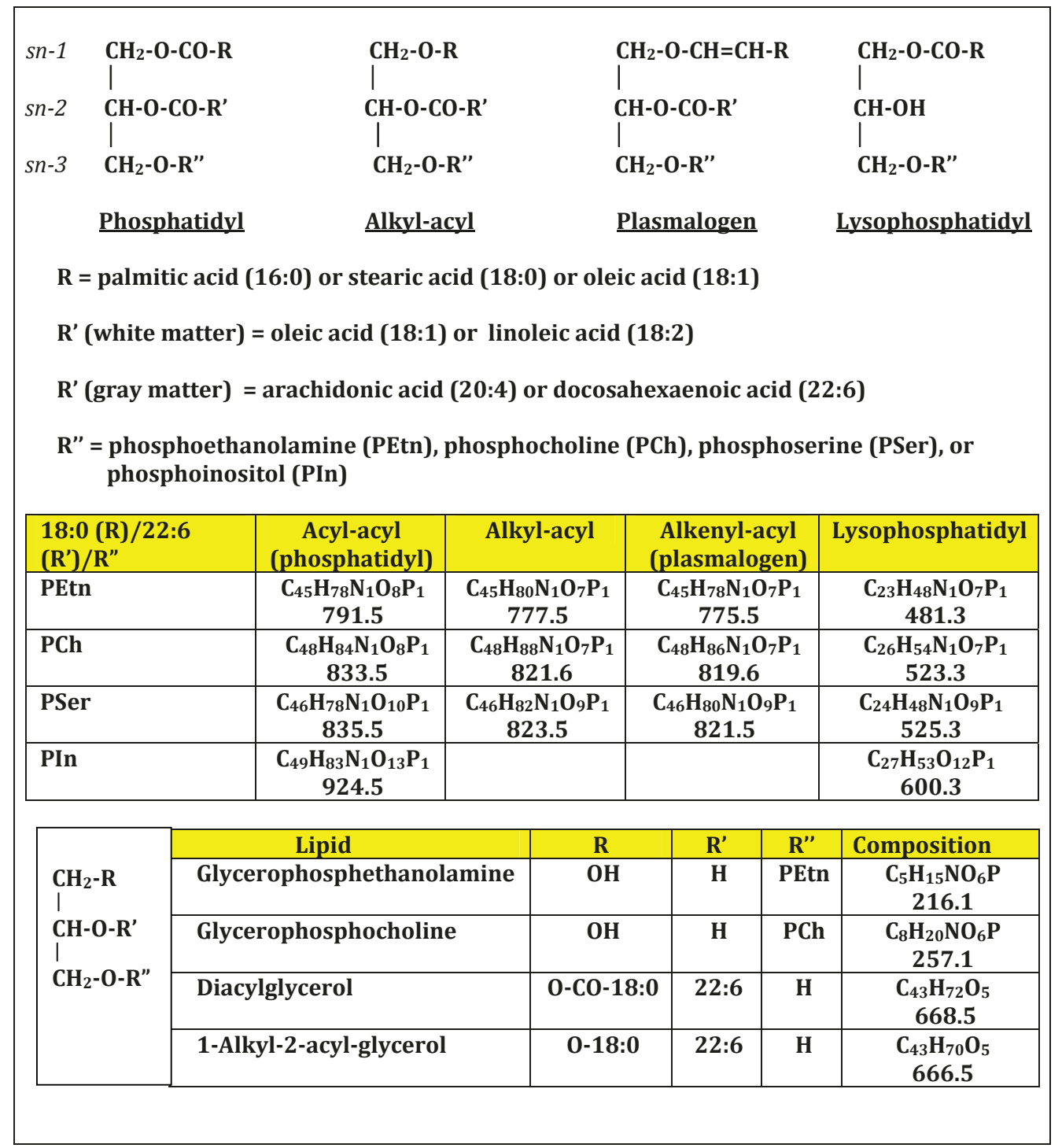

Figure 1. Chemical structures of glycerophospholipids. Phosphatidyl glycerophospholipids are diacyl lipids in which fatty acids are the substituents at sn-1 and sn-2 and charged bases (PEtn, PCh, PSer or PIn) at sn-3. Ether lipids possess a fatty alcohol at sn-1 and a fatty acid at sn-2. In plasmalogens, the fatty alcohol at sn-1 possesses a desaturation at the first two carbons in the fatty alcohol substitution. Loss of a fatty acid generates the lysophosphatidyl glycerophospholipids, via deacylation at sn-2 of diacyl glycerophospholipids.

Parkinson's substantia nigra and demonstrated anatomic specificity, being marked in the mid-temporal cortex but not the cerebellum $[8,9]$. These deficiencies in a major structural phospholipid pool were rapidly validated by other research groups and quantification of individual PlsEtns by tandem mass spectrometry demonstrated that white matter PlsEtns (that is, oleic or linoleic acid at sn-2; Figure 1) were decreased by up to $40 \%$ early in the disease process $[9,10]$. Gray matter PlsEtns (that is, docosahexaenoic (DHA) or arachidonic acid at sn-2; Figure 1) decreased in a disease severity-dependent manner [9]. Of particular note, while there were dramatic decreases in cerebellar white matter PlsEtns, there were no changes in cerebellar gray matter PlsEtns even in late stage AD [9]. PlsEtn changes were also shown to be specific in that phosphatidylethanolamines, serine glycerophospholipids and inositol glycerophospholipids were unaltered [8-10] (nomenclature and structures are presented in Figure 1).

Subsequent research demonstrated disease severitydependent decreases in circulating DHA and PlsEtns, particularly PlsEtns containing DHA at sn-2 [11,12]. While plasma DHA is derived both from the diet and from peroxisome-dependent synthesis by the liver and gastrointestinal epithelium, peroxisomes are obligatory 


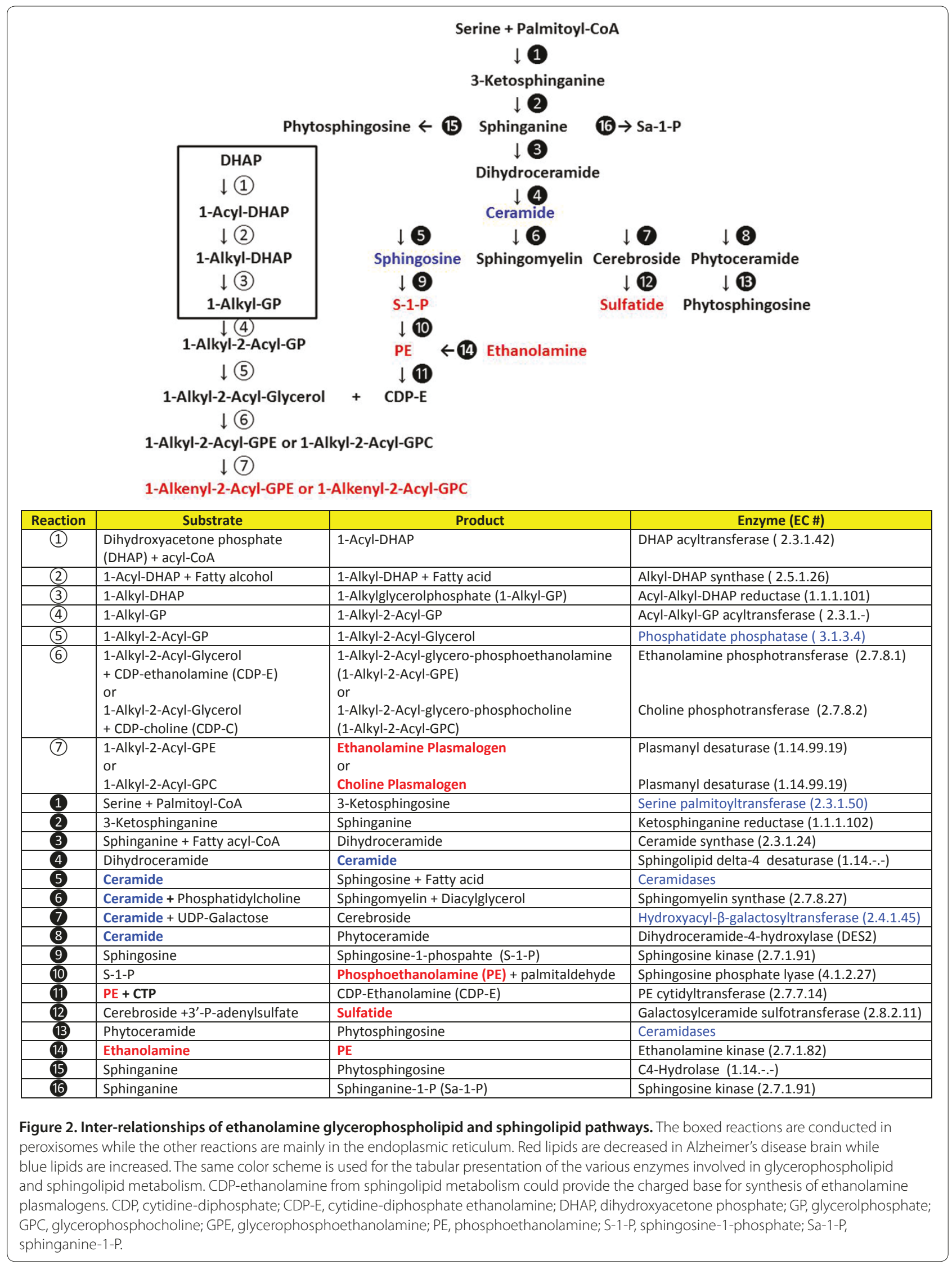


for PlsEtn synthesis [13]. Based on these data, a peroxisomal deficit in $\mathrm{AD}$ was proposed [11] and was soon demonstrated in AD liver [14] and AD brain [15,16]; however, these data do not also preclude a contribution of lipid peroxidation in plasmalogen decrements or potential drug-induced liver toxicity. Brain and liver DHA is decreased in AD tissues and pristanic acid, a metabolite of dietary phytanic acid that is metabolized exclusively by peroxisomes [17], is elevated in AD liver [14]. Decreases in AD brain PlsEtn levels are accompanied by accumulation of very long chain fatty acids (VLCFAs): behenic acid (C22:0), lignoceric acid (C24:0) and hexacosanoic acid (C26:0) [15]. These VLCFAs are all metabolized by peroxisomes $[13,15]$, again supporting peroxisomal dysfunction in AD. Furthermore, the decrements in brain [9], liver [14] and plasma [11,12] DHAcontaining plasmalogens and the accumulation of VLCFAs in the cortex [15] correlate with cognitive deficit in $\mathrm{AD}$ patients. In contrast, decreases in white matter PlsEtns occur early in the disease process and do not correlate with cognitive status [9].

Supply of PlsEtn building blocks to the central nervous system (CNS) is complicated in that a number of lipid transport mechanisms are involved. DHA (omega-3) and arachidonic acid (omega-6), which are long chain polyunsaturated fatty acids essential for PlsEtn synthesis, comprise $8 \%$ and $6 \%$ of brain dry weight, respectively [1]. Fatty acid binding proteins [18] are major determinants of fatty acid transport into and within the CNS. The heart type fatty acid binding protein (FABP3) has been evaluated in $\mathrm{AD}$ and found to be decreased in brain and plasma, but increased in CSF [18]. Epidermal fatty acid binding protein (FABP5) and brain fatty acid binding protein (FABP7), both present in the brain, remain to be evaluated.

Circulating plasmalogens and plasmalogen precursors also gain access to the CNS. Circulating plasmalogens are synthesized mainly by the liver and gastrointestinal epithelium $[14,19]$ and are exported into the circulation via chaperone transport proteins of which low density lipoprotein (LDL) is a major carrier, containing micromolar concentrations of PlsEtns [20]. These chaperone proteins are critical since free plasmalogens are metabolically unstable. In the case of the blood-brain barrier and blood-retinal barrier, transport of the plasmalogens is via an LDL receptor-mediated transcytosis pathway that bypasses the lysosomal compartment [21]. This transport pathway preferentially shuttles LDL enriched in DHAcontaining phospholipids [22]. LDL receptor function in $\mathrm{AD}$ and its potential impact on supply of plasmalogens to the CNS remain to be more clearly defined.

In summary, there are a number of significant decrements in brain polyunsaturated fatty acids and PlsEtns in $\mathrm{AD}$. These include early and dramatic decreases in white matter PlsEtns in the brain and a disease severity-dependent decrease in gray matter PlsEtns. These changes in lipid dynamics appear to be the result of peroxisomal dysfunction in both the liver [14] and brain [15,16], a conclusion further supported by the accumulation of VLCFAs in AD brain [15]. Since circulating plasmalogens are decreased in a number of other clinical conditions, the effects of these potential confounds need to be addressed in future AD studies. These include plasmalogen decrements in ischemic cerebrovascular disease [23], Parkinson's disease [24], hypertension [25], uremia [26], and hyperlipidemia [27].

\section{Choline glycerophospholipids}

Choline is an essential precursor for the synthesis of glycerophosphocholines (GPCs). However, choline levels are affected dramatically by agonal status and postmortem delays in human tissue handling. The net result is that there is no consistent finding from publications of choline levels in AD brain. However, as observed with PlsEtns, it appears there are decrements in brain choline plasmalogens (PlsChs; Figure 1) [28] but not in phosphatidylcholines [10]. These analyses were not performed with liquid chromatography-tandem mass spectrometry such that individual PlsChs were not characterized but fatty acid analyses did demonstrate deficits in the total DHA-containing PlsCh pool [28]. The PlsCh metabolite GPC has been shown to be increased in AD cortex [6] and CSF [29]. The accumulation of GPC is potentially indicative of increased degradation of choline glycerophospholipids and/or decreased GPC metabolism. Decreases in AD cortical phospholipase D [30], an enzyme that removes choline from GPC and lysophosphatidylcholines (LPCs), may also contribute to GPC accumulation. However, GPC phosphodiesterase (EC 3.1.4.2, EC 3.1.4.46), another enzyme that metabolizes GPC, is increased in AD cortex but not cerebellum [31]. In a non-targeted metabolomics study of $\mathrm{AD}$ plasma, decrements in LPC 16:0 (palmitic acid) and LPC 18:2 (linoleic acid) have been reported [32]. The anatomical source(s) of these circulating metabolites remains to be defined.

In summary, it appears that peroxisomal deficits in $\mathrm{AD}$ [14-16] result in decreases in brain PlsChs and alterations in the metabolism of GPCs. The impact of alterations in the metabolism of GPCs on cholinergic neurotransmission remain to be investigated. These early autopsy studies need to be repeated and the individual PlsChs characterized, while studies of plasma LPCs require larger population-based studies.

\section{Glycerophospholipid remodeling}

While brain glycerophospholipids possess almost exclusively palmitic (16:0), stearic (18:0) or oleic acid (18:1) at 
sn- 1 , the fatty acid substitution at sn- 2 is much more varied. In white matter, the sn-2 position is dominated by oleic acid while in gray matter DHA (22:6) and arachidonic acid (20:4) predominate. A further critically important feature of the sn-2 fatty acid substitutions is that they are dynamic, undergoing continuous remodeling. Lipid remodeling is a process involved in the generation of a large family of PlsEtns and PlsChs in the brain. The lipid remodeling pathway involves removal of sn-2 fatty acids by the 2-acyl hydrolases, phospholipase A2 (EC 3.1.1.4) and acylglycerol lipase (EC 3.1.1.23) and reacylation, with alternative fatty acids, by acylCoA:lysophospholipid 2-acyltransferases [33].

Of the 22 different phospholipase A2 (PLA2) enzymes, several have been evaluated in AD. Plasmalogen-selective PLA2 [34] and cytosolic PLA2 [35] have been reported to be increased in AD cortex. $\mathrm{Ca}^{++}$-dependent PLA2 is increased in AD CSF [36], decreased in AD cortex [31], and unaffected in $\mathrm{AD}$ cerebellum [31]. $\mathrm{Ca}^{++}$-independent PLA2 is decreased in both AD CSF [37] and AD cortex [31], and unaffected in AD cerebellum [31]. Clearly, further characterization of changes in the multiple isoforms of PLA2 in AD is required. In parallel with increases in $\mathrm{AD}$ cortical plasmalogen-selective PLA2 [34], lysophospholipid acyltransferase is increased [31], further supporting the tight coupling of deacylationreacylation in lipid remodeling. This coordinated deacylation-reacylation mechanism appears to be located mainly in the endoplasmic reticulum [33], also the location of the final steps of plasmalogen and sphingolipid synthesis (Figure 2).

A major aspect of lipid remodeling that remains to be evaluated in $\mathrm{AD}$ are the signaling cascades potentially evoked by lipids released by deacylation at sn- 2 of glycerophospholipids. In the case of released arachidonic acid, a vast array of eicosanoids can be generated as mediators of neuroinflammation, a common feature of $\mathrm{AD}$ brain [38]. In contrast, released DHA can be utilized in the generation of anti-inflammatory and neuroprotective docosanoids [39], lipid metabolites that remain to be investigated in $\mathrm{AD}$ brain.

In summary, studies of lipid remodeling in AD brain indicate that these processes are augmented and further contribute to changes in glycerophospholipid dynamics in $\mathrm{AD}$ and in the processing of amyloid [40].

\section{Sphingolipids}

Sphingolipids are major structural lipids of CNS membranes and in the case of sulfatides (Figure 3) are highly expressed in myelin. Sphingolipids constitute 5 to $7 \%$ of the myelin lipid pool and are synthesized by oligodendrocytes. Early reports of large decrements in white matter sulfatides [4] were validated and individual sulfatides characterized by tandem mass spectrometry
[41]. The major sulfatide pools in the human CNS include D18:1 (sphingosine)/24:1 (nervonic acid), D18:1/24:1h ( $\alpha$-hydroxynervonic acid; cerebronic acid), and D18:1/26:1 (hexacosenoic acid), (Figure 3). While sulfatide levels are decreased in $\mathrm{AD}$ cortex, the compositional distribution of sulfatide subtypes is unaltered in AD brain [42]. Sulfatide depletion is up to $93 \%$ in the gray matter and occurs early in the disease process while sulfatide depletion is disease severity-dependent in white matter, with up to $58 \%$ depletion [41]. Sulfatide losses in AD cortex are disease specific in that they do not occur in subjects with Parkinson's disease, dementia with Lewy bodies, frontotemporal dementia, or multiple sclerosis [43]. Sulfatide synthesis (Figure 2) does not appear to be altered in that the pivotal synthetic enzyme galactocerebroside sulfotransferase (Figure 2, reaction 12) is normal in $\mathrm{AD}$ postmortem cortex, as are levels of cerebrosides, the direct precursors of sulfatides [10]. In contrast to $\mathrm{AD}$ cortex, recent studies of $\mathrm{AD}$ hippocampus have reported decrements in the total cerebroside pool, while cerebrosides with 2-hydroxylated fatty acids (for example, cerebronic acid) are slightly increased [44]. These changes are specific to the hippocampus and are not seen in the cerebellum [44]. Whether these differences between cortex and hippocampus represent a true regional difference or if the relative mix of white and gray matter sampled is responsible for these differences remains to be investigated.

Decreases in $\mathrm{AD}$ cortical sulfatides are paralleled by large increases in cortical $[41,45,46]$ and CSF [47] ceramides, which are precursors/degradation products of sulfatides (Figure 3). White matter ceramides are increased three-fold in the AD temporal cortex and cerebellum early in the disease process [41]. In late stage $\mathrm{AD}$, these increases in white matter ceramides remain elevated at two-fold that of age-matched controls while gray matter ceramides are unaltered at all stages of AD [41]. Immunohistochemistry studies suggest that the increases in cortical ceramides are mainly in astrocytes [47]. The major ceramide that increases is N24:1 (nervonic acid) ceramide (Figure 3), the precursor/degradation product of the predominant brain sulfatide D18:1 (sphingosine)/24:1 (nervonic acid) [41].

Increases in ceramide levels are paralleled by increases in both acid ceramidase (EC 3.5.1.23) [46,48] and galactosylceramidase (EC 3.2.1.46) [49]. Augmentation of brain ceramidase levels is most likely the basis of increased brain levels of sphingosine (Figure 2, reaction 5) in $\mathrm{AD}$ [49]. In contrast, sphingosine-1-phosphate is decreased in $\mathrm{AD}$ brain [46]. The potential signaling effects of alterations in brain ceramides, sphingosine and sphingosine-1-phosphate remain to be determined. One of the established actions of ceramides is the activation of brain plasmalogen-selective PLA2 [50], thereby potentially 


\begin{tabular}{|c|c|c|c|c|c|}
\hline \multirow{3}{*}{\multicolumn{3}{|c|}{ 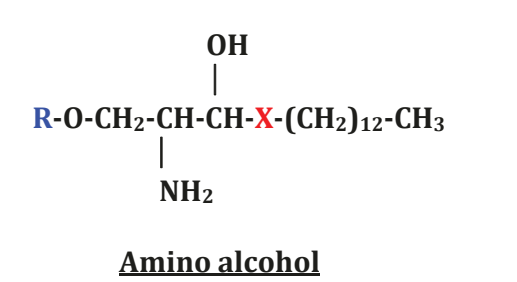 }} & \multirow{2}{*}{\multicolumn{3}{|c|}{$\underset{\mathrm{R}_{\mathrm{NH}}-\mathrm{CO}-\mathrm{CH}_{2}-\mathrm{CH}-\mathrm{CH}-\mathrm{CH}=\mathrm{CH}-\left(\mathrm{CH}_{2}\right)_{12}-\mathrm{CH}_{3}}{\mathrm{OH}}$}} \\
\hline & & & & & \\
\hline & & & \multicolumn{3}{|c|}{ NH-CO-R' } \\
\hline \multicolumn{2}{|l|}{ Amino alcohol } & $\mathbf{R}$ & $\mathrm{X}$ & Formula & MW \\
\hline \multicolumn{2}{|l|}{ Sphinganine } & $\mathbf{H}$ & $\mathrm{CH}_{2}-\mathrm{CH}_{2}$ & $\mathrm{C}_{18} \mathrm{H}_{39} \mathrm{NO}_{2}$ & 301.3 \\
\hline \multicolumn{2}{|c|}{ Phytosphingosine } & $\mathbf{H}$ & $\mathrm{CH}(-\mathrm{OH})-\mathrm{CH}_{2}$ & $\mathrm{C}_{18} \mathrm{H}_{39} \mathrm{NO}_{3}$ & 317.3 \\
\hline \multicolumn{2}{|c|}{ Sphingosine } & $\mathbf{H}$ & $\mathrm{CH}=\mathrm{CH}$ & $\mathrm{C}_{18} \mathrm{H}_{37} \mathrm{NO}_{2}$ & 299.3 \\
\hline \multicolumn{2}{|c|}{ Sphingosine-1-phosphate } & $\mathrm{PO}_{3} \mathrm{H}_{2}$ & $\mathrm{CH}=\mathrm{CH}$ & $\mathrm{C}_{18} \mathrm{H}_{38} \mathrm{NO}_{5} \mathrm{P}$ & 379.2 \\
\hline Sulfatide & \multicolumn{2}{|c|}{$\mathbf{R}$} & $\mathbf{R}^{\prime}$ & Formula & MW \\
\hline D18:1/24:1 & \multicolumn{2}{|c|}{ Galactose-3-sulfate } & Nervonic acid & $\mathrm{C}_{48} \mathrm{H}_{91} \mathrm{NO}_{11} \mathrm{~S}$ & 889.6 \\
\hline D18:1/24:1h & \multicolumn{2}{|c|}{ Galactose-3-sulfate } & Cerebronic acid & $\mathrm{C}_{48} \mathrm{H}_{91} \mathrm{NO}_{12} \mathrm{~S}$ & 905.6 \\
\hline D18:1/26:1 & \multicolumn{2}{|c|}{ Galactose-3-sulfate } & Hexacosenoic acid & $\mathrm{C}_{50} \mathrm{H}_{95} \mathrm{NO}_{12} \mathrm{~S}$ & 933.6 \\
\hline Cerebroside & \multicolumn{2}{|c|}{$\mathbf{R}$} & $\mathbf{R}^{\prime}$ & Formula & MW \\
\hline D18:1/24:1h & \multicolumn{2}{|c|}{ Galactose } & Nervonic acid & $\mathrm{C}_{48} \mathrm{H}_{91} \mathrm{NO}_{8}$ & 809.6 \\
\hline Ceramide & \multicolumn{2}{|c|}{$\mathbf{R}$} & $\mathbf{R}^{\prime}$ & Formula & MW \\
\hline D18:1/18:0 & \multicolumn{2}{|c|}{$-\mathbf{H}$} & Stearic acid & $\mathrm{C}_{36} \mathrm{H}_{71} \mathrm{NO}_{3}$ & 565.5 \\
\hline D18:1/22:0 & \multicolumn{2}{|c|}{$-\mathrm{H}$} & Behenic acid & $\mathrm{C}_{40} \mathrm{H}_{79} \mathrm{NO}_{3}$ & 621.6 \\
\hline D18:1/24:1 & \multicolumn{2}{|c|}{$-\mathbf{H}$} & Nervonic acid & $\mathrm{C}_{42} \mathrm{H}_{81} \mathrm{NO}_{3}$ & 647.6 \\
\hline Sphingomyelin & \multicolumn{2}{|c|}{$\mathbf{R}$} & $\mathbf{R}^{\prime}$ & Formula & MW \\
\hline D18:1/16:0 & \multicolumn{2}{|c|}{ Phosphocholine } & Palmitic acid & $\mathrm{C}_{39} \mathrm{H}_{79} \mathrm{~N}_{2} \mathrm{O}_{6} \mathrm{P}$ & 702.5 \\
\hline D18:1/18:1 & \multicolumn{2}{|c|}{ Phosphocholine } & Oleic acid & $\mathrm{C}_{41} \mathrm{H}_{81} \mathrm{~N}_{2} \mathrm{O}_{6} \mathrm{P}$ & 728.5 \\
\hline
\end{tabular}

Figure 3. Chemical structures of the amino alcohol precursors and their sphingolipid products. MW, molecular weight.

contributing to the decrements in brain plasmalogens in AD (see the 'Choline glycerophospholipids' section). While these studies of AD cortex are consistent in their observations of elevated ceramide levels, analysis of $\mathrm{AD}$ hippocampus has revealed decrements in ceramide levels [44]. Clearly, more detailed regional AD brain analyses are needed as well as more detailed comparisons of mild cognitive impairment (MCI) and AD disease stages.

Plasma lipidomics studies have also demonstrated increased ceramide levels in AD patients. These changes include increases in N16:0 (palmitic acid) and N21:0 (heneicosanoic acid) ceramides [51]. In contrast, another study has reported no differences in plasma ceramide levels in AD [52] but decreased plasma levels in MCI. The major ceramide species that are decreased in $\mathrm{MCI}$ patients contain the long chain saturated fatty acids C22:0 (behenic acid), C24:0 (lignoceric acid), and C26:0 (cerotic acid) [52]. The ceramide precursor sphinganine and its metabolite phytosphinganine (Figure 2; Figure 3) have also been reported to be decreased in AD plasma [32]. All of these studies are based on small patient numbers, and larger population studies are clearly needed to resolve the reported differences.

In contrast to brain, which demonstrates no alterations in sphingomyelin levels [10], decrements in plasma sphingomyelins have been monitored, particularly sphingomyelins with very long chain monounsaturated fatty acid substitutions [51]. These include sphingomyelin D18:1 (sphingosine)/24:1 (nervonic acid) and D18:1/22:1 (erucic acid). In addition, longitudinal studies over a 2.3 year period have demonstrated that higher plasma levels of sphingomyelins are predictors of slower disease progression in AD patients [53].

In summary, brain sphingolipid metabolism is dramatically affected early in AD white and gray matter. Decrements in myelin sulfatides support imaging studies 
demonstrating hypomyelination in $\mathrm{MCI}$ and $\mathrm{AD}$ patients. These sulfatide losses appear to involve both increased degradation and increased export from oligodendrocytes $[42,48]$. Clearly, dramatic alterations in brain sphingolipid metabolism occur early in the pathogenesis of $\mathrm{AD}$. The reliability/utility of reported changes in plasma sphingolipids remain to be validated in larger and more diverse patient populations.

\section{Pathophysiology of glycerophospholipid and sphingolipid changes in AD \\ Myelin and neuronal membrane structural defects}

Myelin is extremely rich in both plasmalogens and sulfatides as structural lipids. PlsEtns encompass $22.4 \%$ of plasmalogens in myelin, in contrast to choline plasmalogens, which comprise only $0.9 \%$ of that mass [13]. In addition to myelin, PlsEtns are major structural phospholipids in neuronal membranes and mitochondria, comprising about $30 \%$ of total phospholipids and $85 \%$ of ethanolamine phospholipids [9]. Sulfatides account for $5 \%$ of myelin lipids and are critical to paranodal junction formation. The combined loss of myelin sulfatides and PlsEtns further reflects a complex and progressive hypomyelination process in $\mathrm{AD}$. This hypomyelination is further demonstrated by decrements in protein biomarkers of myelin integrity, namely 2,3'-cyclic nucleotide-3'-phosphodiesterase [54] and myelin basic protein [55]. Imaging studies in MCI and AD patients have consistently demonstrated microstructural changes in myelin. In particular, decreased myelin integrity appears to be most marked in late-myelinating fiber tracts, such as the inferior- and superior-longitudinal fasciculi [56]. This hypomyelination combined with decreased supply of neuronal plasmalogens also can explain the dramatic shrinkage and dysfunction of AD cortical neurons and the nucleus basalis-cortex cholinergic projection [57-59], which is critical in cognitive function.

\section{Structural lipid deficits and signal transduction}

Sphingolipids and sphingolipid metabolites are important mediators of signal transduction in the CNS, as they act on membrane-associated receptors and are precursors for a number of bioactive lipids. Ceramides are thought to participate in neuroinflammation and in activation of cell death pathways $[41,45]$. Ceramides are known to induce a number of inflammatory cytokines, including IL-6, which is dramatically elevated in AD brain [38]. Deregulation of sphingolipid metabolism may also have dramatic effects on nuclear function. Sphingomyelin is the major sphingolipid in nuclei and is involved in chromatin architecture, RNA stability and DNA synthesis [60]. Sphingosine and sphingosine-1-phosphate, metabolites of sphingomyelin, also regulate gene transcription and histone acetylation, respectively [60]. The impact of alterations in the dynamics of sphingolipids on these functions in AD remains to be investigated.

Plasmalogens are major structural phospholipids in membranes, such that changes in their concentrations and/or composition will dramatically affect membrane fluidity, thereby negatively affecting the functions of membrane transporters, ion channels and membranebound enzymes [13,59]. Alterations in lipid remodeling [33] also will affect signal transduction via alterations in the concentrations of free arachidonic acid and DHA available for the synthesis of eicosanoids and docosanoids, respectively [39].

\section{Structural lipid deficits and cholinergic function}

The cholinergic hypothesis of cognitive dysfunction in $\mathrm{AD}$ remains a core concept in AD research [59]. Understanding the pathophysiology of this neurotransmitter deficit is at the core of many research group's efforts in defining potential new therapeutic approaches for AD. Based upon our current knowledge of lipidomics changes in the AD brain, the following points can be summarized about the possible negative contributions of lipid deficits on cholinergic function.

First, decrements in sulfatides and plasmalogens, resulting in hypomyelination and membrane dysfunction, may be responsible for the marked shrinkage of nucleus basalis-cortical cholinergic neurons [57-59].

Second, hypomyelination is known to reduce axonal transport in AD cortex [61]. Decreased anterograde transport of choline acetyltransferase and the acetylcholine vesicular transporter would reduce the synthetic and storage capacities for acetylcholine in the cortical nerve terminal fields [59]. Decreased retrograde transport of essential trophic factors from the cortex to the cholinergic cell bodies in the basal forebrain would lead to decreased trophic support and decreased cell body synthesis of critical proteins required for cholinergic neuronal function [59].

Third, decreased availability of plasmalogens to cholinergic nerve terminal would impact mitochondrial function and neurotransmitter vesicular fusion with the presynaptic membrane, both resulting in decreased synaptic acetylcholine release [59]. Accumulation of VLCFAs [15] also will negatively affect mitochondrial function.

Fourth, decreased plasmalogens in AD membrane lipid rafts [62] would alter membrane fluidity uncoupling cholinergic muscarinic receptors in AD cortex [63], as has been shown to occur in plasmalogen-deficient cell lines [64]. Decreased acetylcholine release coupled with impaired postsynaptic signal transduction may well be responsible for the marked cholinergic deficit in AD patients [59].

Fifth, deficits in sulfatides and plasmalogens also will negatively affect gap junction integrity, thereby impairing 
glial metabolic support of cholinergic nerve terminals in the cortex [59].

Sixth, decreases in membrane plasmalogen levels result in decreased export of cholesterol, further contributing to membrane rigidity $[59,65]$. This increase in membrane rigidity is hypothesized to contribute to abnormal processing of $\beta$-amyloid in $\mathrm{AD}[59,65]$.

Seventh, neuroinflammation is a hallmark feature of $\mathrm{AD}$ brain $[38,59]$. In this regard, neuroinflammation has been demonstrated to be induced by metabolites of sphingolipids and by decrements in membrane plasmalogens, both features of AD brain [59].

Eighth, astroglial-neuronal and neuronal-neuronal exchange of exosomes may be altered since these secretory vesicles have a lipid composition similar to lipid rafts, being rich in cholesterol, sphingomyelin and plasmalogens. Decreases in these communication pathways [66] may also negatively affect cholinergic neurotransmission.

\section{Conclusion}

It is now clear that peroxisomal dysfunction in $\mathrm{AD}$ leads to deficits in both ethanolamine and choline plasmalogens and accumulation of VLCFAs. These alterations have the potential to be major determinants in neuronal dysfunction in AD. Alterations in the dynamics of sphingolipids are just becoming more understood and presumably underlie the myelin abnormalities that have been reported in AD. Integrating lipidomics findings to date, it appears that in white matter there are about 58\% depletions in sulfatides, three-fold increases in ceramides and 40\% decreases in PlsEtns early in the disease process (Clinical Dementia Rating of 0.5) and that these changes are sustained in advanced disease (Clinical Dementia Rating of 3.0) [9,10]. In contrast, sulfatides are depleted by $93 \%$, ceramides are unaltered and PlsEtns demonstrate a disease severity-dependent decrease in $\mathrm{AD}$ gray matter $[9,41]$. These data indicate that alterations in glycerophospholipids and sphingolipids occur early in the $\mathrm{AD}$ process and probably involve a number of factors related to lipid synthesis, transport and degradation.

The challenges ahead are to evaluate plasma lipidomics in larger and more diverse patient populations to determine their utility in disease diagnosis and in monitoring disease progress. More detailed regional lipidomics analysis of AD brain samples is required as well as indepth comparisons at different disease stages. A further comparison that has high probability of yielding very valuable data will be to investigate the lipidomics of brain samples from individuals with no cognitive impairment demonstrating significant AD neuropathological burden [67]. Understanding the differences in lipidomics between no-cognitive-impairment and AD brains will help pinpoint critical metabolic changes that result in cholinergic dysfunction. These approaches will define the utility of glycerophospholipids and sphingolipids as potential biomarkers of disease progression and help define new points of pharmacological intervention for the treatment of $\mathrm{AD}$.

\section{Abbreviations \\ AD, Alzheimer's disease; ApoE, apolipoprotein E; CNS, central \\ nervous system; CSF, cerebrospinal fluid; DHA, docosahexaenoic \\ acid; GPC, glycerophosphocholine; LDL, low density lipoprotein; LPC, lysophosphocholine; $\mathrm{MCl}$, mild cognitive impairment; PLA2, phospholipase A2; PlsCh, choline plasmalogen; PIsEtn, ethanolamine plasmalogen; VLCFA, very long chain fatty acid.}

Competing interests

The author declares that he has no competing interests.

\section{Acknowledgements}

I wish to thank my many collaborators who have worked with me over the last 30 years on $A D$ research.

Published: 1 February 2012

\section{References}

1. Florent-Béchard S, Desbène C, Garcia P, Allouche A, Youssef I, Escanyé MC, Koziel V, Hanse M, Malaplate-Armand C, Stenger C, Kriem B, Yen-Potin FT, Olivier JL, Pillot T, Oster T: The essential role of lipids in Alzheimer's disease. Biochimie 2009, 91:804-809.

2. Astarita G, Piomelli D: Towards a whole-body systems [multi-organ] lipidomics in Alzheimer's disease. Prostaglandins Leukot Essent Fatty Acids 2011, 85:197-203.

3. Di Paolo G, Kim TW: Linking lipids to Alzheimer's disease: cholesterol and beyond. Nat Rev Neurosci 2011, 12:284-296.

4. Wallin A, Gottfries CG, Karlsson I, Svennerholm L: Decreased myelin lipids in Alzheimer's disease and vascular dementia. Acta Neurol Scand 1989, 80:319-323.

5. Ellison DW, Beal MF, Martin JB: Phosphoethanolamine and ethanolamine are decreased in Alzheimer's disease and Huntington's disease. Brain Res 1987, 417:389-392.

6. Nitsch RM, Blusztajn JK, Pittas AG, Slack BE, Growdon JH, Wurtman RJ: Evidence for a membrane defect in Alzheimer disease brain. Proc Natl Acad SciUSA 1992, 89:1671-1675.

7. Molina JA, Jiménez-Jiménez FJ, Vargas C, Gómez P, de Bustos F, Ortí-Pareja M, Tallón-Barranco A, Benito-León J, Arenas J, Enríquez-de-Salamanca R: Cerebrospinal fluid levels of non-neurotransmitter amino acids in patients with Alzheimer's disease. J Neural Transm 1998, 105:279-286.

8. Ginsberg L, Rafique S, Xuereb JH, Rapoport SI, Gershfeld NL: Disease and anatomic specificity of ethanolamine plasmalogen deficiency in Alzheimer's disease brain. Brain Res 1995, 698:223-226.

9. Han X, Holtzman DM, McKeel DW Jr: Plasmalogen deficiency in early Alzheimer's disease subjects and in animal models: molecular characterization using electrospray ionization mass spectrometry. J Neurochem 2001, 77:1168-1180.

10. Han X: Lipid alterations in the earliest clinically recognizable stage of Alzheimer's disease: implication of the role of lipids in the pathogenesis of Alzheimer's disease. Curr Alzheimer Res 2005, 2:65-77.

11. Goodenowe DB, Cook LL, Liu J, Lu Y, Jayasinghe DA, Ahiahonu PW, Heath D, Yamazaki Y, Flax J, Krenitsky KF, Sparks DL, Lerner A, Friedland RP, Kudo T, Kamino K, Morihara T, Takeda M, Wood PL: Peripheral ethanolamine plasmalogen deficiency: a logical causative factor in Alzheimer's disease and dementia. J Lipid Res 2007, 48:2485-2498.

12. Wood PL, Mankidy R, Ritchie S, Heath D, Wood JA, Flax J, Goodenowe DB: Circulating plasmalogen levels and Alzheimer Disease Assessment ScaleCognitive scores in Alzheimer patients. J Psychiatry Neurosci 2010, 35:59-62.

13. Wallner S, Schmitz G: Plasmalogens the neglected regulatory and scavenging lipid species. Chem Phys Lipids 2011, 164:573-589.

14. Astarita G, Jung KM, Berchtold NC, Nguyen VQ, Gillen DL, Head E, Cotman CW, Piomelli D: Deficient liver biosynthesis of docosahexaenoic acid correlates with cognitive impairment in Alzheimer's disease. PLoS One 2010, 5:e12538.

15. Kou J, Kovacs GG, Höftberger R, Kulik W, Brodde A, Forss-Petter S, 
Hönigschnabl S, Gleiss A, Brügger B, Wanders R, Just W, Budka H, Jungwirth S, Fischer P, Berger J: Peroxisomal alterations in Alzheimer's disease. Acta Neuropathol 2011, 122:271-283.

16. Grimm MO, Kuchenbecker J, Rothhaar TL, Grösgen S, Hundsdörfer B, Burg VK, Friess P, Müller U, Grimm HS, Riemenschneider M, Hartmann T: Plasmalogen synthesis is regulated via alkyl-dihydroxyacetonephosphate-synthase by amyloid precursor protein processing and is affected in Alzheimer's disease. J Neurochem 2011, 116:916-925.

17. Wanders RJ, Komen J, Ferdinandusse S: Phytanic acid metabolism in health and disease. Biochim Biophys Acta 2011, 1811:498-507.

18. Owada Y: Fatty acid binding protein: localization and functional significance in the brain. Tohoku J Exp Med 2008, 214:213-220.

19. Roels F, Espeel M, Pauwels M, De Craemer D, Egberts HJ, van der Spek P: Different types of peroxisomes in human duodenal epithelium. Gut 1991, 32:858-865.

20. Wiesner P, Leidl K, Boettcher A, Schmitz G, Liebisch G: Lipid profiling of FPLCseparated lipoprotein fractions by electrospray ionization tandem mass spectrometry. J Lipid Res 2009, 50:574-585.

21. Candela P, Gosselet F, Miller F, Buee-Scherrer V, Torpier G, Cecchelli R, Fenart L : Physiological pathway for low-density lipoproteins across the bloodbrain barrier: transcytosis through brain capillary endothelial cells in vitro. Endothelium 2008, 15:254-264.

22. Polozova A, Gionfriddo E, Salem N Jr : Effect of docosahexaenoic acid on tissue targeting and metabolism of plasma lipoproteins. Prostaglandins Leukot Essent Fatty Acids 2006, 75:183-190.

23. Hoerrmann W, Donis J, Sluga E, Stütz H, Paltauf F: Serum plasmalogens in ischemic cerebrovascular disease. Vasa 1991, 20:319-322.

24. Dragonas C, Bertsch T, Sieber CC, Brosche T: Plasmalogens as a marker of elevated systemic oxidative stress in Parkinson's disease. Clin Chem Lab Med 2009, 47:894-897.

25. Graessler J, Schwudke D, Schwarz PE, Herzog R, Shevchenko A, Bornstein SR: Top-down lipidomics reveals ether lipid deficiency in blood plasma of hypertensive patients. PLoS One 2009, 4:e6261.

26. Brosche T, Platt D, Knopf B: Decreased concentrations of serum phospholipid plasmalogens indicate oxidative burden of uraemic patients undergoing haemodialysis. Nephron 2002, 90:58-63.

27. Brosche T: Plasmalogen levels in serum from patients with impaired carbohydrate or lipid metabolism and in elderly subjects with normal metabolic values. Arch Gerontol Geriatr 2001, 32:283-294.

28. Igarashi M, Ma K, Gao F, Kim HW, Rapoport SI, Rao JS: Disturbed choline plasmalogen and phospholipid fatty acid concentrations in Alzheimer's disease prefrontal cortex. J Alzheimers Dis 2011, 24:507-517.

29. Walter A, Korth U, Hilgert M, Hartmann J, Weichel O, Hilgert M, Fassbender K, Schmitt A, Klein J: Glycerophosphocholine is elevated in cerebrospinal fluid of Alzheimer patients. Neurobiol Aging 2004, 25:1299-1303.

30. Kanfer JN, Hattori H, Orihel D: Reduced phospholipase D activity in brain tissue samples from Alzheimer's disease patients. Ann Neurol 1986, 20:265-267.

31. Ross BM, Moszczynska A, Erlich J, Kish SJ: Phospholipid-metabolizing enzymes in Alzheimer's disease: increased lysophospholipid acyltransferase activity and decreased phospholipase A2 activity. J Neurochem 1998, 70:786-793.

32. Li NJ, Liu WT, Li W, Li SQ, Chen XH, Bi KS, He P: Plasma metabolic profiling of Alzheimer's disease by liquid chromatography/mass spectrometry. Clin Biochem 2010, 43:992-997.

33. Shindou H, Shimizu T: Acyl-CoA:lysophospholipid acyltransferases. J Biol Chem 2009, 284:1-5.

34. Farooqui AA: Studies on plasmalogen-selective phospholipase A2 in brain. Mol Neurobiol 2010, 41:267-273.

35. Stephenson DT, Lemere CA, Selkoe DJ, Clemens JA: Cytosolic phospholipase A2 (CPLA2) immunoreactivity is elevated in Alzheimer's disease brain. Neurobiol Dis 1996, 3:51-63.

36. Chalbot S, Zetterberg H, Blennow K, Fladby T, Grundke-lqbal I, Iqbal K: Cerebrospinal fluid secretory Ca2+-dependent phospholipase A2 activity is increased in Alzheimer disease. Clin Chem 2009, 55:2171-2179.

37. Smesny S, Stein S, Willhardt I, Lasch J, Sauer H: Decreased phospholipase A2 activity in cerebrospinal fluid of patients with dementia. J Neural Transm 2008, 115:1173-1179.

38. Wood JA, Wood PL, Ryan R, Graff-Radford NR, Pilapil C, Robitaille Y, Quirion R Cytokine indices in Alzheimer's temporal cortex: no changes in mature IL-1 beta or IL-1RA but increases in the associated acute phase proteins
IL-6, alpha 2-macroglobulin and C-reactive protein. Brain Res 1993, 629:245-252.

39. Niemoller TD, Bazan NG: Docosahexaenoic acid neurolipidomics. Prostaglandins Other Lipid Mediat 2010, 91:85-89.

40. Sanchez-Mejia RO, Mucke L: Phospholipase A2 and arachidonic acid in Alzheimer's disease. Biochim Biophys Acta 2010, 1801:784-790.

41. Han X, M Holtzman D, McKeel DW Jr, Kelley J, Morris JC: Substantial sulfatide deficiency and ceramide elevation in very early Alzheimer's disease: potential role in disease pathogenesis. J Neurochem 2002, 82:809-818.

42. Yuki D, Sugiura Y, Zaima N, Akatsu H, Hashizume Y, Yamamoto T, Fujiwara M, Sugiyama K, Setou M: Hydroxylated and non-hydroxylated sulfatide are distinctly distributed in the human cerebral cortex. Neuroscience 2011, 193:44-45.

43. Cheng H, Xu J, McKeel DW Jr, Han X: Specificity and potential mechanism of sulfatide deficiency in Alzheimer's disease: an electrospray ionization mass spectrometric study. Cell Mol Biol 2003, 49:809-818.

44. Hejazi L, Wong JW, Cheng D, Proschogo N, Ebrahimi D, Garner B, Don AS: Mass and relative elution time profiling: two-dimensional analysis of sphingolipids in Alzheimer's disease brains. Biochem J 2011, 438:165-175

45. Cutler RG, Kelly J, Storie K, Pedersen WA, Tammara A, Hatanpaa K, Troncoso JC, Mattson MP: Involvement of oxidative stress-induced abnormalities in ceramide and cholesterol metabolism in brain aging and Alzheimer's disease. Proc Nat Acad Sci U S A 2004, 101:2070-2075.

46. He X, Huang Y, Li B, Gong CX, Schuchman EH: Deregulation of sphingolipid metabolism in Alzheimer's disease. Neurobiol Aging 2010, 31:398-408.

47. Satoi $\mathrm{H}$, Tomimoto $\mathrm{H}$, Ohtani R, Kitano T, Kondo T, Watanabe M, Oka N, Akiguchi I, Furuya S, Hirabayashi Y, Okazaki T: Astroglial expression of ceramide in Alzheimer's disease brains: a role during neuronal apoptosis. Neuroscience 2005, 130:657-666.

48. Huang Y, Tanimukai H, Liu F, lqbal K, Grundke-lqbal I, Gong CX: Elevation of the level and activity of acid ceramidase in Alzheimer's disease brain. Eur J Neurosci 2004, 20:3489-3497.

49. Katsel P, Li C, Haroutunian V: Gene expression alterations in the sphingolipid metabolism pathways during progression of dementia and Alzheimer's disease: a shift toward ceramide accumulation at the earliest recognizable stages of Alzheimer's disease? Neurochem Res 2007, 32:845-856.

50. Latorre E, Collado MP, Fernández I, Aragonés MD, Catalán RE: Signaling events mediating activation of brain ethanolamine plasmalogen hydrolysis by ceramide. Eur J Biochem 2003, 270:36-46.

51. Han X, Rozen S, Boyle SH, Hellegers C, Cheng H, Burke JR, Welsh-Bohmer KA, Doraiswamy PM, Kaddurah-Daouk R: Metabolomics in early Alzheimer's disease: identification of altered plasma sphingolipidome using shotgun lipidomics. PLoS One 2011, 6:e21643.

52. Mielke MM, Haughey NJ, Ratnam Bandaru W, Schech S, Carrick R, Carlson MC, Mori S, Miller MI, Ceritoglu C, Brown T, Albert M, Lyketsos CG: Plasma ceramides are altered in mild cognitive impairment and predict cognitive decline and hippocampal volume loss. Alzheimers Dement 2010, 6:378-385.

53. Mielke MM, Haughey NJ, Bandaru W, Weinberg DD, Darby E, Zaidi N, Pavlik V, Doody RS, Lyketsos CG: Plasma sphingomyelins are associated with cognitive progression in Alzheimer's disease. J Alzheimers Dis 2011. 27:259-269.

54. VIkolinský R, Cairns N, Fountoulakis M, Lubec G: Decreased brain levels of 2,3'-cyclic nucleotide-3'-phosphodiesterase in Down syndrome and Alzheimer's disease. Neurobiol Aging 2001, 22:547-553.

55. Wang DS, Bennett DA, Mufson EJ, Mattila P, Cochran E, Dickson DW: Contribution of changes in ubiquitin and myelin basic protein to agerelated cognitive decline. Neurosci Res 2004, 48:93-100.

56. Stricker NH, Schweinsburg BC, Delano-Wood L, Wierenga CE, Bangen KJ, Haaland KY, Frank LR, Salmon DP, Bondi MW: Decreased white matter integrity in late-myelinating fiber pathways in Alzheimer's disease supports retrogenesis. Neuroimage 2009, 45:10-16.

57. Perry RH, Candy JM, Perry EK, Irving D, Blessed G, Fairbairn AF, Tomlinson BE: Extensive loss of choline acetyltransferase activity is not reflected by neuronal loss in the nucleus of Meynert in Alzheimer's disease. Neurosci Lett 1982, 33:311-315.

58. Etienne P, Robitaille $Y$, Wood $P$, Gauthier $S$, Nair NP, Quirion R: Nucleus basalis neuronal loss, neuritic plaques and choline acetyltransferase activity in advanced Alzheimer's disease. Neuroscience 1986, 19:1279-1291.

59. Wood PL, Khan MA, Mankidy R, Smith T, Goodenowe DB: Plasmalogen deficit: A new and testable hypothesis for the etiology of Alzheimer's 
disease. In Alzheimer's Disease Pathogenesis - Core Concepts, Shifting Paradigms and Therapeutic Targets. Edited by De La Monte S. InTech; 2011

[http://www.intechopen.com/articles/show/title/

plasmalogen-deficit-a-new-and-testable-hypothesis-for-the-etiology-ofalzheimer-s-disease]

60. Luki NC, Sewr MB: Nuclear sphingolipid metabolism. Annu Rev Physio/ 2012, 74:1.1-1.21

61. Dai J, Buijs RM, Kamphorst W, Swaab DF: Impaired axonal transport of cortical neurons in Alzheimer's disease is associated with neuropathological changes. Brain Res 2002, 948:138-144.

62. Martín V, Fabelo N, Santpere G, Puig B, Marín R, Ferrer I, Díaz M: Lipid alterations in lipid rafts from Alzheimer's disease human brain cortex. J Alzheimers Dis 2010, 19:489-502.

63. Tsang SW, Lai MK, Kirvell S, Francis PT, Esiri MM, Hope T, Chen CP, Wong PT: Impaired coupling of muscarinic $\mathrm{M} 1$ receptors to $\mathrm{G}$-proteins in the neocortex is associated with severity of dementia in Alzheimer's disease. Neurobiol Aging 2006, 27:1216-1223.

64. Périchon R, Moser AB, Wallace WC, Cunningham SC, Roth GS, Moser HW: Peroxisomal disease cell lines with cellular plasmalogen deficiency have impaired muscarinic cholinergic signal transduction activity and amyloid precursor protein secretion. Biochem Biophys Res Commun 1998, 248:57-61.

65. Mankidy R, Ahiahonu PW, Ma H, Jayasinghe D, Ritchie SA, Khan MA, Su-Myat KK, Wood PL, Goodenowe DB: Membrane plasmalogen composition and cellular cholesterol regulation: a structure activity study. Lipids Health Dis 2010, 9:62-79.

66. Smalheiser NR: Exosomal transfer of proteins and RNAs at synapses in the nervous system. Biol Direct 2007, 2:35.

67. Erten-Lyons D, Woltjer RL, Dodge H, Nixon R, Vorobik R, Calvert JF, Leahy M, Montine T, Kaye J: Factors associated with resistance to dementia despite high Alzheimer disease pathology. Neurology 2009, 72:354-360.

doi:10.1186/alzrt103

Cite this article as: Wood PL: Lipidomics of Alzheimer's disease: current status. Alzheimer's Research \& Therapy 2012, 4:5 